\title{
Model to describe light scattering by polymer film containing droplets with inhomogeneous anchoring of liquid crystal molecules at the polymer-droplet interface: asymmetry effect in the angular distribution of light
}

\author{
A. V. Konkolovich ${ }^{\mathrm{a}}$, A. A. Miskevich ${ }^{\mathrm{a}}$, M. N. Krakhalev ${ }^{\mathrm{b}, \mathrm{c}}$, O. O. Prishchepa ${ }^{\mathrm{b}, \mathrm{c}}$, A. V. Shabanov ${ }^{\mathrm{b}}$, \\ V. Ya. Zyryanov ${ }^{\mathrm{b}}$, and V. A. Loiko ${ }^{\mathrm{a}}$ \\ ${ }^{a}$ Stepanov Institute of Physics, National Academy of Sciences of Belarus, Minsk, Belarus \\ ${ }^{b}$ Federal Research Center - Krasnoyarsk Scientific Center, Siberian Branch, Russian Academy of \\ Sciences, Kirensky Institute of Physics, Krasnoyarsk, Russia \\ ${ }^{c}$ Institute of Engineering Physics and Radio Electronics, Siberian Federal University, Krasnoyarsk, \\ Russia \\ *E-mail: loiko@ifanbel.bas-net.by
}

\begin{abstract}
A model to describe light scattering by polymer film containing of monolayer of liquid crystal droplets with inhomogeneous anchoring of liquid crystal molecules at the polymerdroplet interface is developed. It is based on the interference approximation of the wave scattering theory. The director field distribution in the droplet volume is determined by solving the free energy density minimization problem using the relaxation method. The spatial distribution of droplets in the layer is described by the hard disks model. The amplitude scattering matrices of individual droplets are found in the anomalous diffraction approximation. The algorithm for numerical analysis of the characteristics of light scattered in a polymer film containing droplets at homogeneous and inhomogeneous surface anchoring is described in terms of the partial filling factors of the monolayer film. Electrically controllable symmetry breaking effect of angular distribution of light scattered by films containing droplets with inhomogeneous anchoring at the polymer-droplet interface is described and experimentally confirmed.
\end{abstract}

Keywords: Polymer-dispersed liquid crystal film; liquid crystal droplets; light transmission; angular distribution of scattered light

\section{INTRODUCTION}

Polymer-dispersed liquid crystal (PDLC) films are composite materials consisting of liquidcrystal droplets dispersed in a polymer matrix $[1,2]$. The electrically, magnetically, thermally, and preasure induced change in orientation of the liquid crystal molecule results in variation of the film optical properties. Such films can be flexible. They possess high mechanical strength, light resistance, have a simple production technology and are used in photonics and optoelectronics. On the basis of these films it is possible to create: displays, intensity and phase modulators, polarizers and polarization converters, lenses, filters, reflectors, etc. [3-16]. 
At present there are two approaches to control the electro-optical response of the PDLC films in the scattering mode.

The first one is based on the classic Freedericksz effect [17-19]. In this case the surface anchoring of the liquid crystal (LC) molecules with the polymer matrix remains unchanged with changing the control electric field. The director configuration in the droplet returns to its original state due to the elastic forces in the film when the field is switched off. Classic Freedericksz effect underlies the operation of modern electro-optical liquid crystal devices.

Another approach is based on the local Freedericksz transition [20,21]. In this case the local change in the orientation of the optical axes (directors) in the LC droplet volume is due to changes of LC molecule anchoring at the droplet-polymer interface. Modification of the interface anchoring can be achieved using the ionic surfactants driven by the applied electric field [22]. The ionic surfactant creates heterogeneity of interfacial anchoring and transforms the droplet director configuration.

Theoretical description of the electrooptical response of the PDLC films with non-uniform interphase anchoring has to take into account the optical anisotropy of the liquid crystal, the orientation of the LC molecules in the droplets (which depends on the surface anchoring at the interface), concentration of droplets, the optical characteristics of the polymer matrix and liquid crystal, and other parameters [2, 8-11].

At the moment, there is no rigorous theory of light scattering by dispersed optically anisotropic medium. Because of this, the problem of light propagation is solved by the approximate methods [23-28]. In this paper, to describe light scattering in the monolayer films consisting of liquid crystal droplets embedded in polymer matrix the intrerferece approximation and the model of hard discs are used. To analyse small-angle light scattering by a single LC droplet we use anomalous diffraction approximation. Configuration of the director field (director configuration) in a droplet is determined on the base of the free energy minimization problem solution using the relaxation method. We have analyzed small-angle scattering of light by a single LC droplet and by the PDLC film. The model of hard discs is discussed in detailes in application to the considered problem. It is studied the electro-optical effect of breaking the symmetry of the small-angle distribution of scattered radiation in films with inhomogeneous surface anchcoring of liquid crystal molecules at the polymer-droplet surface. The effect consists in the fact that in the scattering plane (the plane defined by the wave vectors of the incident and scattered waves) the values of intensities of radiation scattered at angle $+\theta_{s}$ and angle $-\theta_{s}$ (see Fig.1) are different relative to the illumination direction. Some preliminary data on the effect are given in [29-31]. It is worth noting that in films with homogeneous surface anchoring there is a symmetrical distribution of the radiation intensity scattered at the angles $+\theta_{s}$ and $-\theta_{s}[1,2,29-31]$. 


\section{OPTICAL MODEL TO DESCRIBE SMALL-ANGLE LIGHT SCATTERING BY A PDLC FILM}

Let us consider a PDLC monolayer illuminated by a linearly polarized plane wave with wave vector directed normally to the monolayer plane. The geometry of illumination is presented in Fig.1. In here $x y z$ is the laboratory coordinate system associated with the propagation direction of the incident wave ( $x$ axis) and the plane of the monolayer $(y, z) ; A$ is the area of the investigated part of the layer; $\mathbf{E}_{i}$ and $\mathbf{k}_{i}$ are the polarization vector and the wave vector of the incident wave; $\mathbf{k}_{s}$ is the wave vector of the scattered wave; $\theta_{s}$ is the scattering angle; $\varphi_{s}$ is the angle of orientation of the scattering plane $\left(\mathbf{k}_{i}, \mathbf{k}_{s}\right)$; vectors $\mathbf{N}_{j}$ are the optical axes (directors) of the LC droplets (they characterize the average orientation direction of the long axes of LC molecules in the volume of the droplet $[1,2,32]$ with number $j(j=1,2, \ldots, N ; N$ is the number of droplets $)$.

FIG. 1. Geometry of the PDLC monolayer illumination and light scattering. Notations are in the text.

The layer consists of monodisperse or polydisperse droplets in the form of spheres or spheroids with circular cross sections in the plane $(y, z)$ of the monolayer. We analyze the $v v$ - and $v h$ - components of the scattered light, which are polarized parallel and orthogonal to the 
polarization plane $\left(\mathbf{E}_{i}, \mathbf{k}_{i}\right)$ of the incident wave, respectively. In Fig. 1 they are indicated by the lines $v v$ and $v h$.

Using the interference approximation [33,34], we write the expressions for $v v$ - and $v h$ components of the scattered field at the observation point $M$ (in the far field zone) with radius vector $\mathbf{R}$ as follows [35]:

$$
E_{v v, v h}^{s}(\mathbf{R})=\sum_{j=1}^{N} E_{j}^{v v, v h}(\mathbf{R}) \exp \left(-i \mathbf{k}_{s} \mathbf{r}_{j}\right)=\sum_{l=1}^{m} E_{l}^{v v, v h}(\mathbf{R}) \sum_{j=1}^{N_{l}} \exp \left(-i \mathbf{k}_{s} \mathbf{r}_{j}^{l}\right),
$$

where $E_{j}^{v v, v h}(\mathbf{R})$ are components of the field created by the $j$-th droplet into the point $\mathbf{R}, \mathbf{r}_{j}$ is radius vector of the droplet center in the monolayer plane $(y, z), N_{l}$ is the number of the $l$-type droplets (droplets with the same area of cross section by the $(y, z)$ plane), $m$ is the number of types of droplets.

The $v v$ - and $v h$ - components of the scattered field $E_{l}{ }^{v, v h}(\mathbf{R})$ are:

$$
E_{l}^{v v, v h}(\mathbf{R})=f_{l}^{v v, v h}\left(\mathbf{k}_{s}\right) \frac{i E_{i}}{k R} \exp (i k R),
$$

where $f_{l}^{v v, v h}\left(\mathbf{k}_{\mathrm{s}}\right)$ are the $v v$ - and $v h$ - components of the vector amplitude scattering function [33,36] of the individual droplet of type $l, E_{i}$ is the amplitude of the incident wave, $k=2 \pi n_{p} / \lambda, n_{p}$ is the refractive index of polymer matrix, $\lambda$ is the wavelength of incident light, $R=|\mathbf{R}|$.

To determine $v v$ - and $v h$ - components of the intensity of the incoherently (diffusely) scattered radiation $I_{v v}^{i n c}$ and $I_{v h}^{i n c}$ we use the equation [34]:

$$
I_{v v, v h}^{i n c}=\left\langle\left|E_{v v, v h}^{s}(\mathbf{R})\right|^{2}\right\rangle_{\mathbf{r}_{j}}-\left|\left\langle E_{v v, v h}^{s}(\mathbf{R})\right\rangle_{\mathbf{r}_{j}}\right|^{2},
$$

where the angle brackets $\langle\ldots\rangle$ denote averaging over the droplet positions $\mathbf{r}_{j}$. Below, if the variable of averaging is not indicated, the angle brackets denote averaging over the droplet sizes.

Assuming that the spatial distribution of the LC droplets is statistically homogeneous and isotropic, using Eqs. (1) - (3), the $I_{v v}{ }^{i n c}$ and $I_{v h}{ }^{i n c}$ components [33] can be written as:

$$
I_{v v, v h}^{i n c}\left(\theta_{s}, \varphi_{s}\right)=C \frac{\eta}{k^{2}\langle\sigma\rangle} \sum_{l, l^{\prime}=1}^{m}\left(P_{l} P_{l^{\prime}}\right)^{1 / 2} f_{l}^{v v, v h}\left(\theta_{s}, \varphi_{s}\right) f_{l^{\prime}}^{* v v, v h}\left(\theta_{s}, \varphi_{s}\right) S_{l l^{\prime}}\left(\theta_{s}\right),
$$

where $C=A E_{i}{ }^{2} / R^{2} ; \eta=N\langle\sigma\rangle / A$ is the filling factor of the PDLC monolayer equal to the ratio of the cross section area of the droplets by the monolayer plane to the area where they are distributed; $\langle\sigma\rangle$ is the average value of the droplet projections to the monolayer plane $(y, z) ; P_{l}=N_{l} / N$ and $P_{l^{\prime}}=N_{l^{\prime}} / N$ are the fractions of droplet types $l$ and $l^{\prime} ; \sum_{l=1}^{m} P_{l}=1 ; l, l^{\prime}=1,2, \ldots, m ;$ symbol $*$ denotes complex conjugation; functions $S_{l l}\left(\theta_{s}\right)$ are the partial structure factors [37]. They are determined as follows:

$$
S_{l l^{\prime}}\left(\theta_{s}\right)=\delta_{l l^{\prime}}+8\left(\eta_{l} \eta_{l^{\prime}}\right)^{1 / 2} \int_{0}^{\infty}\left(W_{l l^{\prime}}(u)-1\right) J_{0}\left(2 k\left(c_{l} c_{l^{\prime}}\right)^{1 / 2} u \sin \theta_{s}\right) u d u
$$


where $\delta_{l l^{\prime}}$ is the Kronecker symbol $\left(\delta_{l l^{\prime}}=1\right.$ for $l=l^{\prime}$ and $\delta_{l l^{\prime}}=0$ for $\left.l \neq l^{\prime}\right) ; \eta_{l}=N_{l} \pi c_{l}^{2} / A, \eta_{l^{\prime}}=N_{l^{\prime}} \pi c_{l^{\prime}}^{2} / A$ are the partial filling factors; $c_{l}$ and $c_{l^{\prime}}$ are radii of the $y z$-sections of droplets of $l$ and $l^{\prime}$ types; $W_{l l^{\prime}}(u)$ are the partial radial distribution functions [37]; $J_{0}$ is the zero-order cylindrical Bessel function of the first kind.

The partial structure factors $S_{l l^{\prime}}\left(\theta_{S}\right)$ can be calculated using a generalized Ornstein-Zernike integral equation [38-40]. To determine $S_{l l^{\prime}}\left(\theta_{s}\right)$ we use the relation [41]:

$$
\hat{S}\left(\theta_{s}\right)=\left(\hat{I}-\hat{\widetilde{C}}\left(\theta_{s}\right)\right)^{-1}
$$

Here $\hat{S}\left(\theta_{s}\right)$ is the matrix of the partial structure factors $S_{l l}\left(\theta_{s}\right) ; \hat{I}$ is the unit matrix of dimension $m \times m ; \hat{\widetilde{C}}\left(\theta_{s}\right)$ is the matrix of the 2D Fourier transforms of the direct partial correlation functions $\widetilde{C}_{l l^{\prime}}\left(\theta_{s}\right)$.

In this paper we use analytical approximation [41], obtained by the solution of the OrnsteinZernike equation in the Percus-Yevick [37] approximation for hard disks:

$$
\widetilde{C}_{l l^{\prime}}\left(\theta_{s}\right)=\left(\eta_{l} \eta_{l^{\prime}}\right)^{1 / 2} \widetilde{c}_{l l^{\prime}}\left(\theta_{s}\right)
$$

where

$$
\begin{gathered}
\eta_{l, l^{\prime}}=P_{l, l^{\prime}} \frac{c_{l, l^{\prime}}^{2}}{\left\langle c^{2}\right\rangle} \eta, \\
\widetilde{c}_{l l^{\prime}}\left(\theta_{s}\right)=-\left\{\begin{array}{l}
\chi_{l l^{\prime}}^{(2)} \frac{2 J_{1}\left(k c_{l} \sin \theta_{s}\right)}{k c_{l} \sin \theta_{s}} \frac{2 J_{1}\left(k c_{l^{\prime}} \sin \theta_{s}\right)}{k c_{l^{\prime}} \sin \theta_{s}}+\chi_{l}^{(1)} \frac{2 J_{1}\left(k c_{l} \sin \theta_{s}\right)}{k c_{l} \sin \theta_{s}} 2 J_{0}\left(k c_{l^{\prime}} \sin \theta_{s}\right)+ \\
+\chi_{l^{\prime}}^{(1)} \frac{2 J_{1}\left(k c_{l^{\prime}} \sin \theta_{s}\right)}{k c_{l^{\prime}} \sin \theta_{s}} 2 J_{0}\left(k c_{l} \sin \theta_{s}\right)++\chi_{l l^{\prime}}^{(0)} \frac{2 J_{1}\left(k\left(c_{l}+c_{l^{\prime}}\right) \sin \theta_{s}\right)}{k\left(c_{l}+c_{l^{\prime}}\right) \sin \theta_{s}}
\end{array}\right\},
\end{gathered}
$$

$J_{1}$ is the first-order cylindrical Bessel function of the first kind,

$$
\begin{gathered}
\chi_{l l^{\prime}}^{(0)}=\frac{\left(c_{l}+c_{l^{\prime}}\right)^{2}}{c_{l} c_{l^{\prime}}} \frac{1}{1-\eta}, \\
\chi_{l, l^{\prime}}^{(1)}=\frac{c_{l, l^{\prime}}\langle c\rangle}{\left\langle c^{2}\right\rangle} \frac{2 \eta}{(1-\eta)^{2}}, \\
\chi_{l l^{\prime}}^{(2)}=\frac{c_{l} c_{l^{\prime}}}{\left\langle c^{2}\right\rangle} \frac{\eta}{(1-\eta)^{2}}+\frac{c_{l} c_{l^{\prime}}\langle c\rangle^{2}}{\left\langle c^{2}\right\rangle^{2}} \frac{2 \eta^{2}}{(1-\eta)^{3}}, \\
\langle c\rangle=\sum_{l=1}^{m} P_{l} c_{l}, \\
\left\langle c^{2}\right\rangle=\sum_{l=1}^{m} P_{l} c_{l}^{2} .
\end{gathered}
$$


For monolayer of monodisperse spherical droplets or spheroidal droplets with the circular cross-section in the monolayer plane the equality $c_{l}=c_{l}=c$ takes place, where $c$ is radius of the droplet projection to the monolayer plane, and equations (4) are transformed as follows:

$$
I_{v v, v h}^{i n c}\left(\theta_{s}, \varphi_{s}\right)=C \frac{\eta}{\sigma k^{2}}\left|f_{v v, v h}\left(\theta_{s}, \varphi_{s}\right)\right|^{2} S\left(\theta_{s}\right) .
$$

Here

$$
S\left(\theta_{s}\right)=\left\{1+\frac{4 \eta}{1-\eta} \frac{2 J_{1}(2 u)}{2 u}+\frac{4 \eta^{2}}{(1-\eta)^{2}} J_{0}(u) \frac{2 J_{1}(u)}{u}+\left(\frac{\eta^{2}}{(1-\eta)^{2}}+\frac{2 \eta^{3}}{(1-\eta)^{3}}\right)\left[\frac{2 J_{1}(u)}{u}\right]^{2}\right\}^{-1}
$$

is the structure factor [42] of monolayer of monodisperse droplets; $\sigma=\pi c^{2} ; u=k c \sin \theta_{s} ; \eta=N \sigma A$.

Analytical formulas (7)-(14) and (16) for the structure factors can be used for monolayers with filling factors up to $\sim 0.7[33,37]$.

At $\eta \leq 0.4$ it is possible to analyze angular distribution of light scattered by the PDLC monolayer using the substitution model $[37,43,44]$. It allows one to simplify essentially the problem solution.

In this model the partial structure factors $S_{l l}\left(\theta_{s}\right)$ (Eqs. 5) can be written as:

$$
S_{l l^{\prime}}\left(\theta_{s}\right)=\left\{\begin{array}{l}
0, l \neq l^{\prime} \\
\left\langle S\left(\theta_{s}\right)\right\rangle, l=l^{\prime}
\end{array} .\right.
$$

Here $\left\langle S\left(\theta_{s}\right)\right\rangle$ is the structure factor of monolayer of monodisperse droplets (Eq. (15)) averaged over the droplet radius $c$ :

$$
\left\langle S\left(\theta_{s}\right)\right\rangle=\sum_{l=1}^{m} P_{l} S\left(\theta_{s}, c_{l}\right)
$$

Then the $v v$-and $v h$-components are:

$$
I_{v v, v h}^{i n c}\left(\theta_{s}, \varphi_{s}\right)=C \frac{\eta}{\langle\sigma\rangle k^{2}}\left\{\left\langle\left|f_{v v, v h}\left(\theta_{s}, \varphi_{s}\right)\right|^{2}\right\rangle_{c, \mathbf{N}_{j}}+\left|\left\langle f_{v v, v h}\left(\theta_{s}, \varphi_{s}\right)\right\rangle_{c, \mathbf{N}_{j}}\right|^{2}\left[\left\langle S\left(\theta_{s}\right)\right\rangle-1\right]\right\} .
$$

The last equation can be rewritten as:

$$
I_{v v, v h}^{i n c}\left(\theta_{s}, \varphi_{s}\right)=C \frac{\eta}{\langle\sigma\rangle k^{2}}\left\{\left\langle\left\langle\left. f_{v v, v h}\left(\theta_{s}, \varphi_{s}\right)\right|^{2}\right\rangle_{c, \mathbf{N}_{j}}+\left|\left\langle f_{v v, v h}\left(\theta_{s}, \varphi_{s}\right)\right\rangle_{c, \mathbf{N}_{j}}\right|^{2}\left\langle S\left(\theta_{s}\right)\right\rangle\right\},\right.
$$

where

$$
\left\langle\left|\Delta f_{v v, v h}\left(\theta_{s}, \varphi_{s}\right)\right|^{2}\right\rangle_{c, \mathbf{N}_{j}}=\left\langle\left|f_{v v, v h}\left(\theta_{s}, \varphi_{s}\right)\right|^{2}\right\rangle_{c, \mathbf{N}_{j}}-\left|\left\langle f_{v v, v h}\left(\theta_{s}, \varphi_{s}\right)\right\rangle_{c, \mathbf{N}_{j}}\right|^{2}
$$

Numerical data for $I_{v v, v h}^{i n c}\left(\theta_{s}, \varphi_{s}\right)$ obtained by the substitution model are in good agreement with the experimental ones for the monolayer of liquid crystal droplets [45]. 
Note that the substitution model implies that inversion of any two particles does not change the spatial configuration of the entire ensemble. This imposes restrictions on the concentration and the polydispersity degree of droplets.

To analyze $I_{v v}{ }^{s}\left(\theta_{s}, \varphi_{s}\right)$ and $I_{v h}{ }^{s}\left(\theta_{s}, \varphi_{s}\right)$ intensities of radiation scattered by a single LC droplet, write them, using Eq. (2), as follows:

$$
I_{v v, v h}^{s}\left(\theta_{s}, \varphi_{s}\right)=C \frac{1}{\sigma k^{2}}\left|f_{v v, v h}\left(\theta_{s}, \varphi_{s}\right)\right|^{2} .
$$

Here $C=\sigma E_{i}^{2} / R^{2}$.

The $f_{v v, v h}\left(\theta_{s}, \varphi_{s}\right)$ functions of the single LC droplet are defined by the elements $S_{j}(j=1,2,3,4)$ of the amplitude scattering matrix [24,33]:

$$
\begin{aligned}
f_{v v}\left(\theta_{s}, \varphi_{s}\right)= & S_{2}\left(\theta_{s}, \varphi_{s}\right) \cos ^{2}\left(\alpha-\varphi_{s}\right)+S_{1}\left(\theta_{s}, \varphi_{s}\right) \sin ^{2}\left(\alpha-\varphi_{s}\right)+ \\
& \frac{1}{2}\left(S_{3}\left(\theta_{s}, \varphi_{s}\right)+S_{4}\left(\theta_{s}, \varphi_{s}\right)\right) \sin 2\left(\alpha-\varphi_{s}\right) \\
f_{v h}\left(\theta_{s}, \varphi_{s}\right)= & S_{3}\left(\theta_{s}, \varphi_{s}\right) \sin ^{2}\left(\alpha-\varphi_{s}\right)-S_{4}\left(\theta_{s}, \varphi_{s}\right) \cos ^{2}\left(\alpha-\varphi_{s}\right)+ \\
& \frac{1}{2}\left(S_{2}\left(\theta_{s}, \varphi_{s}\right)-S_{1}\left(\theta_{s}, \varphi_{s}\right)\right) \sin 2\left(\alpha-\varphi_{s}\right)
\end{aligned}
$$

where $\alpha$ is the polarization angle of the incident light (Fig. 1).

At illumination by unpolarized light the relations for intensity $I_{n p}{ }^{s}\left(\theta_{s}, \varphi_{s}\right)$ of light, scattered by the monolayer $\left(\operatorname{Inp}^{i n c}\left(\theta_{s}, \varphi_{s}\right)\right)$ and the single $\operatorname{droplet}\left(I_{n p}{ }^{s}\left(\theta_{s}, \varphi_{s}\right)\right.$ are:

$$
I_{n p}^{i n c, s}\left(\theta_{s}, \varphi_{s}\right)=\frac{1}{2}\left[\left(I_{v v}^{i n c, s}\left(\theta_{s}, \varphi_{s}\right)+I_{v h}^{i n c, s}\left(\theta_{s}, \varphi_{s}\right)\right)_{\alpha=0}+\left.\left(I_{v v}^{i n c, s}\left(\theta_{s}, \varphi_{s}\right)+I_{v h}^{i n c, s}\left(\theta_{s}, \varphi_{s}\right)\right)\right|_{\alpha=\pi / 2}\right] .
$$

They are derived from Eqs. (4), (15), (19), (22) with taking into account Eqs. (23), (24) and averaging over the polarization angle $\alpha$ of the incident wave at uniform distribution of light intensity in the range from 0 to $2 \pi$.

To determine elements of the scattering amplitude matrix $S_{j}(j=1,2,3,4)$ of the nematic liquid crystal droplets we used the anomalous diffraction approximation [46,47]. The elements are written below:

$$
\begin{aligned}
& S_{1}\left(\theta_{s}, \varphi_{s}\right)=\frac{k^{2} \sigma}{2 \pi} \int_{\sigma}\left(1-T_{1}(\xi)\right) \exp \left(-i \mathbf{k}_{s} \xi\right) d \xi \\
& S_{2}\left(\theta_{s}, \varphi_{s}\right)=\frac{k^{2} \sigma}{2 \pi} \cos \theta_{s} \int_{\sigma}\left(1-T_{2}(\xi)\right) \exp \left(-i \mathbf{k}_{s} \xi\right) d \xi \\
& S_{3}\left(\theta_{s}, \varphi_{s}\right)=-\frac{k^{2} \sigma}{2 \pi} \cos \theta_{s} \int_{\sigma} T_{3}(\xi) \exp \left(-i \mathbf{k}_{s} \xi\right) d \xi \\
& S_{4}\left(\theta_{s}, \varphi_{s}\right)=-\frac{k^{2} \sigma}{2 \pi} \int_{\sigma} T_{4}(\xi) \exp \left(-i \mathbf{k}_{s} \xi\right) d \xi
\end{aligned}
$$


Here $\mathbf{k}_{s} \boldsymbol{\xi}=\left(k y \cos \varphi_{s}+k z \sin \varphi_{s}\right) \sin \theta_{s}, \mathbf{k}_{s}=\left(k \cos \theta_{s}, k \sin \theta_{s} \cos \varphi_{s}, k \sin \theta_{s} \sin \varphi_{s}\right) ; \boldsymbol{\xi}=(0, y, z)$ is the radius vector in the section $\sigma$ of the droplet by the monolayer plane $(y, z) ; T_{j}(\xi)(j=1,2,3,4)$ are elements of the $2 \times 2$ Jones matrix $\hat{T}(\xi)[48,49]$ of the equivalent amplitude-phase screen.

The Jones matrix $\hat{T}(\xi)$ depends on the internal structure of LC droplets. It is defined as follows:

$$
\hat{T}(y, z)=\left(\begin{array}{ll}
T_{2}(y, z) & T_{3}(y, z) \\
T_{4}(y, z) & T_{1}(y, z)
\end{array}\right)=\prod_{x=x_{\text {ipp }}(y, z)}^{x_{\text {out }}(y, z)} R^{T}(x) P R(x) .
$$

where $x_{\text {inp }}=-\varepsilon a \sqrt{1-\left(y^{2}+z^{2}\right) / c^{2}}$ and $x_{\text {out }}=+\varepsilon a \sqrt{1-\left(y^{2}+z^{2}\right) / c^{2}}$ are the input and output coordinates of the wave front at surface of the LC droplet (Fig. 2a); $\varepsilon=a / \mathrm{c}$ is the anisometry parameter equal to the ratio of the minor axis $a$ of a droplet (directed along the axis $x$ ) to the major axis $c$ (situated in the monolayer plane); $P$ is the matrix describing the local phase shifts for the extraordinary and ordinary waves; $R(x)$ and $R^{T}(x)$ are the coordinate transformation matrices along the path of local bases:

$$
\begin{aligned}
& P=\left(\begin{array}{cc}
\exp \left(i k\left(n_{e}(\mathbf{r}) / n_{p}-1\right) \Delta x\right) & 0 \\
0 & \exp \left(i k\left(n_{o} / n_{p}-1\right) \Delta x\right)
\end{array}\right), \\
& R(x)=\left(\begin{array}{cc}
\cos \left(\varphi(\mathbf{r})-\varphi_{s}\right) & -\sin \left(\varphi(\mathbf{r})-\varphi_{s}\right) \\
\sin \left(\varphi(\mathbf{r})-\varphi_{s}\right) & \cos \left(\varphi(\mathbf{r})-\varphi_{s}\right)
\end{array}\right), \\
& R^{T}(x)=\left(\begin{array}{cc}
\cos \left(\varphi(\mathbf{r})-\varphi_{s}\right) & \sin \left(\varphi(\mathbf{r})-\varphi_{s}\right) \\
-\sin \left(\varphi(\mathbf{r})-\varphi_{s}\right) & \cos \left(\varphi(\mathbf{r})-\varphi_{s}\right)
\end{array}\right) .
\end{aligned}
$$

FIG. 2. Schematic representation of (a) an LC droplet and (b) its volume element $\Delta V$. $\mathbf{n}(\mathbf{r})$ is the local director of the elementary volume $\Delta V$ with radius vector $\mathbf{r} ; x_{i n p}$ and $x_{o u t}$ are the input and output coordinates of the wavefront at the droplet surface; $\mathbf{k}_{i}$ is the wavevector of the incident wave; $\theta$ and $\varphi$ are the polar and azimuthal angles of the local director $\mathbf{n}(\mathbf{r})$ orientation; $\mathbf{e}_{o}$ and $\mathbf{e}_{e}$ are the unit polarization vectors of the ordinary and extraordinary waves in the elementary volume $\Delta V . n_{x}$, $n_{y}, n_{z}$ are the Cartesian coordinates of the local director $\mathbf{n}(\mathbf{r})$.

In Eqs. (31) - (33), $n_{e}(\mathbf{r})$ is the local refractive index of the elementary volume $\Delta V$ (the orientation structure of the liquid crystal in this volume is assumed to be homogeneous and is determined only by the molecular order parameter [1,2]) of the LC droplet with radius vector $\mathbf{r}$ for the extraordinary wave, polarized along the unit vector $\mathbf{e}_{e}$ (see Figure $2 \mathrm{~b}$ ), $n_{o}$ is the local refractive index for the ordinary wave polarized along the unit vector $\mathbf{e}_{o}$, which does not depend on the 
coordinates $x, y, z$ and is equal to the ordinary refractive index $n_{\perp}$ of liquid crystal, $\Delta x$ is the linear size of the elementary volume $\Delta V$ along the direction of illumination, $\varphi(\mathbf{r})$ is the azimuthal angle of the local main plane orientation,

$$
\begin{aligned}
n_{e}(\mathbf{r})= & n_{\|} n_{\perp} / \sqrt{n_{\|}^{2} \cos ^{2} \theta(\mathbf{r})+n_{\perp}^{2} \sin ^{2} \theta(\mathbf{r})}=n_{\|} n_{\perp} / \sqrt{n_{\|}^{2} n_{x}^{2}+n_{\perp}^{2}\left(1-n_{x}^{2}\right)} \\
& \cos \left(\varphi(\mathbf{r})-\varphi_{s}\right)=\left(n_{y} \cos \varphi_{s}+n_{z} \sin \varphi_{s}\right) / \sqrt{1-n_{x}^{2}} \\
& \sin \left(\varphi(\mathbf{r})-\varphi_{s}\right)=\left(n_{z} \cos \varphi_{s}-n_{y} \sin \varphi_{s}\right) / \sqrt{1-n_{x}^{2}}
\end{aligned}
$$

$\theta(\mathbf{r})$ is polar angle of the local director $\mathbf{n}(\mathbf{r})$ (Fig. 2b.), $n_{\|}$is the extraordinary refractive index of the liquid crystal, $n_{x}, n_{y}, n_{z}$ are the Cartesian components of the local director $\mathbf{n}(\mathbf{r})$ at the point with radius-vector $\mathbf{r}$.

To calculate distribution of the local director $\mathbf{n}(\mathbf{r})$ in the volume of nematic LC droplet we used equation for the bulk density of the free energy $F$ in the one-constant approximation $[1,2,50,51]$ :

$$
F=F_{e l}+F_{e},
$$

where

$$
F_{e l}=\frac{1}{2} K\left\{(\operatorname{div} \mathbf{n}(\mathbf{r}))^{2}+(\operatorname{rot} \mathbf{n}(\mathbf{r}))^{2}\right\}
$$

is the energy density of the elastic deformations,

$$
F_{e}=\frac{1}{2} \varepsilon(\mathbf{E} \cdot \mathbf{n}(\mathbf{r}))^{2}
$$

is the electric part of the free energy density, $\mathbf{E}$ is the external control electric field vector, $K$ is the average value of the modulus of elasticity, $\varepsilon^{=} \varepsilon_{0} \Delta \varepsilon, \varepsilon_{0}=8,85 \times 10^{-12} \mathrm{C}^{2} /\left(\mathrm{Nm}^{2}\right)$ is the electric constant, $\Delta \varepsilon$ is the dielectric anisotropy of LC.

When calculating the spatial orientation structure of $\mathbf{n}(\mathbf{r})$ inside the droplet (director configuration) with nonuniform surface anchoring, the contribution of the electric control field to the free energy density $F_{e}$ can be neglected [22,52,53] due to shielding of the external field by the electric field of the spatially separated surfactant ions. Then, for the distribution of the local director $\mathbf{n}(\mathbf{r})$ corresponding to the minimum of free energy density [23], one can write on the basis of the expression (38):

$$
K \Delta \mathbf{n}(\mathbf{r})=0
$$

Here $\Delta$ is the Laplace operator.

To find $n_{x}, n_{y}$, and $n_{z}$ components of the local director $\mathbf{n}(\mathbf{r})$, we used the difference scheme of calculations [28]. 
We use the parameter $w$ to characterize the parts of the droplet surface with normal and tangential anchoring (Fig. 3).

FIG. 3. Schematic representation of the cross section of the LC droplet with inhomogeneous anchoring by the $(x, y)$ plane. Parameter $w$ characterizes fraction of the droplet surface with a normal (homeotropic) surface anchoring; $a$ and $c$ are the semiminor and semimajor axes of the droplet; $\mathbf{N}$ is the droplet optical axis; $\mathbf{k}_{i}$ and $\mathbf{k}_{s}$ are the wave vectors of the incident and scattered waves; $-\theta_{s}$ and $+\theta_{s}$ are the scattering angles. The dashes along the ellips show orientation of the long axes of LC molecules at the droplet surface.

The values of $w=0 \%$ and $w=100 \%$ correspond to the homogeneous anchoring at the LC polymer interface. At $w=0 \%$, only tangential surface anchoring occurs all over the surface and a bipolar director configuration in the droplet is implemented (Fig. 4a).

FIG. 4. Calculated director field distribution in the $y z$ cross section of spherical LC droplet for: (a) bipolar configuration; (b) radial configuration; (c) configuration for droplet with inhomogeneous surface anchoring at $w=50 \%$. Director field distribution is shown by dashes.

At $w=100 \%$, only normal surface anchoring and the radial internal structure of the droplet are realized (Fig. 4b). For other values of $w$ the director configuration in the droplet is more complicated [52,53] (Fig. 4c). Parameter $w$ depends on the external controlling field [28]. By changing the field one can change the intradroplet director field and optical characteristics of the PDLC layer.

The optical axis $\mathbf{N}$ of droplet (Fig. 3) is directed orthogonally to the plane separating the droplet on the two parts with different types of anchoring. Directions $+\mathbf{N}$ and $-\mathbf{N}$ for droplets with inhomogeneous anchoring are not equivalent because the surface anchoring depends on the electric field vector $\mathbf{E}$ orientation [22]. If one changes the vector $+\mathbf{E}$ to $-\mathbf{E}$, the normal anchoring is changed to the tangential one and vice versa. Note that for droplets with tangential anchoring (bipolar configuration of the local director in a droplet), directions $+\mathbf{N}$ and $-\mathbf{N}$ are equivalent (there is no difference in the director configuration in the droplet for fields $+\mathbf{E}$ and $-\mathbf{E}$ ) [2]. It is worth paying attention that the liquid crystal droplet with inhomogeneous surface anchoring is an example of the Janus particles [54-58] with electrically-controllable characteristics. 
Consider the results for angular distribtion of radiation scattered by a single droplet and by a monolayer of droplets, at small scattering angles $\theta_{s}$.

Figure 5 illustrates the angular distribution of light scattered by a single spherical LC droplet with radius $c=5 \mu \mathrm{m}$, at zero polarization angle $(\alpha=0)$ of the incident light, and zero azimuthal scattering angle $\left(\varphi_{s}=0\right)$ for the $v v$-component $\left(I_{v v}{ }^{s}\left(\theta_{s}\right)\right)$.

FIG. 5. Angular dependence of scattered light intensity $I_{v v}{ }^{s}\left(\theta_{s}\right)$ calculated for single spherical droplet at parameter $w=0,25,50,75$, and 100\%. The droplet radius $c=5 \mu \mathrm{m} . \alpha=0^{\circ}, \varphi_{s}=0^{\circ} . n_{\|}=1.717$, $n_{\perp}=1.531(\lambda=0.633 \mu \mathrm{m}), n_{p}=n_{\perp}$.

The calculations are performed for different values of parameter $w$. The results of the calculations shown in Fig. 5, as well as the other ones presented in this section, are fulfilled for the liquid crystal 5CB. At wavelength $\lambda=0.633 \mu \mathrm{m}$ the ordinary refractive index of this LC $n_{\perp}=1.531$, the extraordinary one $n_{\|}=1.717$. The optical anisotropy of the liquid crystal $\Delta n=n_{\|}-n_{\perp}=0.186$. The refractive index of the polymer matrix in calculations was assumed to be equal to the ordinary refractive index of LC: $n_{\perp}=n_{p}$. The values of the intensity of scattered light are presented in arbitrary units. One can see that at $w=0 \%$ and $w=100 \%$, the angular distributions of the scattered light intensity are symmetric with respect to scattering angle $\theta_{s}$. This is the case for any polarization angles $\alpha$ of the incident light and the azimuthal scattering angles $\varphi_{s}$ [29]. The values $w=0 \%$ and $w=100 \%$ correspond to uniform surface anchoring for droplets with bipolar and radial configurations of local director $\mathbf{n}(\mathbf{r})$, respectively. In the case of nonuniform anchoring $(0<w$ $<100 \%$ ) the values of intensities of radiation scattered at angles $+\theta_{s}$ and $-\theta_{s}$ (see Fig.1) are different. I.e. the asymmetry effect with respect to the scattering angle $\theta_{s}$ is observed. The strongest shift of the main peak is observed for $w=50 \%$, when the fractions of normal and tangential anchoring on the surface of a LC droplet are equal.

Figure 6 shows scattered light intensity component $I_{v v}{ }^{s}\left(\theta_{s}\right)$ for $w=50 \%$ at different values of $\varphi_{s}$. One can see that: (i) the asymmetry effect disappears for $\varphi_{s}=45^{\circ}, 90^{\circ}$, and $135^{\circ}$; (ii) intensity distributions for $\varphi_{s}=45^{\circ}$ and $\varphi_{s}=135^{\circ}$ coincide; (iii) the distributions for $\varphi_{s}=0^{\circ}$ and $\varphi_{s}=180^{\circ}$ are specularly symmetric relative to direction of the incident light. Orientation of the scattering plane at which the asymmetry disappears depends on the $w$ parameter. 
FIG. 6. Angular dependence of scattered light intensity $I_{v v}{ }^{s}\left(\theta_{s}\right)$ for single spherical LC droplet at $\varphi_{s}$ $=0,45,90,135,180^{\circ}$. Polarization angle of the incident light $\alpha=0^{\circ} . n_{\|}=1.717, n_{\perp}=1.531 \quad(\lambda=0.633$ $\mu \mathrm{m}), n_{p}=n_{\perp}, w=50 \%, c=5 \mu \mathrm{m}$.

The angular distributions of intensity $I_{v v}{ }^{i n c}\left(\theta_{s}\right)$ of light scattered by an ensemble of LC droplets with inhomogeneous surface anchoring are illustrated by Figs. 7, 8. The results for different values of filling factors $\eta$ (Fig.7) and orientation angles $\varphi_{s}$ of the scattering plane (Fig. 8) are shown. The calculations are performed using the relation (15).

FIG. 7. Angular dependence of intensity $I_{v v}{ }^{i n c}\left(\theta_{s}\right)$ of light scattered by a monolayer of monodisperse spherical LC droplets at filling factor $\eta=0.1,0.3,0.5 . c=5 \mu \mathrm{m}, w=50 \%, \alpha=0^{\circ}, \varphi_{s}=0^{\circ}, n_{\|}=1.717$, $n_{\perp}=1.531(\lambda=0.633 \mu \mathrm{m}), n_{p}=n_{\perp}$.

FIG. 8. Angular dependence of intensity $I_{v v}{ }^{i n c}\left(\theta_{s}\right)$ of light scattered by a monolayer of monodisperse spherical LC droplets at $\varphi_{s}=0,45,90,135,180^{\circ} . \alpha=0^{\circ}, n_{\|}=1.717, n_{\perp}=1.531(\lambda=0.633 \mu \mathrm{m}), n_{p}=n_{\perp}$, $c=5 \mu \mathrm{m}, w=50 \%, \eta=0.5$.

From Fig. 7 it follows that the asymmetry effect increases with increasing the concentration (filling factor $\eta$ ) of liquid crystal droplets. At certain values of size and concentration, it becomes more pronounced due to interference effects at scattering in particulate monolayer. The interference redistribution of the intensity of the scattered light, which affects the degree of manifestation of the asymmetry effect, is determined by the ordering of the system. It depends on the structure factor $S\left(\theta_{s}\right)$. The $I_{v v}{ }^{i n c}\left(\theta_{s}\right)$ component of light scattered by the PDLC monolayer (Fig. 8) has the same features as the $I_{v v}{ }^{s}\left(\theta_{s}, \varphi_{s}\right)$ component for a single droplet (Fig. 6): the asymmetry effect disappears at $\varphi_{s}=45^{\circ}, 90^{\circ}, 135^{\circ}$; dependences $I_{v v}{ }^{i n c}\left(\theta_{s}\right)$ are the same for $\varphi_{s}=45^{\circ}, 135^{\circ}$. They are mirror-symmetric at $\varphi_{s}=0^{\circ}, 180^{\circ}$.

Angular distributions of the $v v$ - and $v h$-components of the light intensity $I_{v v}{ }^{s}\left(\theta_{s}, \varphi_{s}\right), I_{v v}{ }^{i n c}\left(\theta_{s}\right.$, $\left.\varphi_{s}\right)$, and $I_{v h}{ }^{s}\left(\theta_{s}, \varphi_{s}\right), I_{v h}^{i n c}\left(\theta_{s}, \varphi_{s}\right)$ scattered by a single droplet, and by the PDLC monolayer are shown in Fig. 9 and 10. The results are presented for the scattering angles $0^{\circ} \leq \varphi_{s} \leq 360^{\circ}$ and $-8^{\circ} \leq \theta_{s} \leq 8^{\circ}$ and illumination by wave with polarized radiation at polarization angle $\alpha=0$. The lighter areas correspond to a greater intensity. It is clearly seen from the figures that there is no central symmetry for layers (and droplets) with homogeneous anchoring ( $w=0 \%$ и $w=100 \%)$. 
FIG. 9. Calculated $I_{v v}{ }^{s}\left(\theta_{s}, \varphi_{s}\right)$ scattering patterns for the single droplet (a), (c), (e) and $I_{v v}{ }^{i n c}\left(\theta_{s}\right.$, $\left.\varphi_{s}\right)$ scattering patterns for the PDLC monolayer of monodisperse spherical droplets (b), (d), (f). (a), (b): $w=0 \%$. (c), (d): $w=100 \%$. (e), (f): $w=50 \%$. $c=5 \mu \mathrm{m}, \eta=0.5, n_{\|}=1.717, n_{\perp}=1.531$ ( $\left.\lambda=0.633 \mu \mathrm{m}\right)$, $n_{p}=n_{\perp} . y=\sin \theta_{s} \cos \varphi_{s}, z=\sin \theta_{s} \sin \varphi_{s},-8^{\circ} \leq \theta_{s} \leq 8^{\circ}, 0^{\circ} \leq \varphi_{s} \leq 360^{\circ}$. Illumination by linearly polarized plane wave with polarization angle $\alpha=0^{\circ}$.

FIG. 10. Calculated $I_{v h}^{s}\left(\theta_{s}, \varphi_{s}\right)$ scattering patterns for the single droplet (a), (c), (e) and $I_{v h}^{i n c}\left(\theta_{s}, \varphi_{s}\right)$ scattering patterns for the PDLC monolayer of monodisperse spherical droplets (b), (d), (f). (a), (b): $w=0 \%$. (c), (d): $w=100 \%$. (e), (f): $w=50 \%$. $c=5 \mu \mathrm{m}, \eta=0.5, n_{\|}=1.717, n_{\perp}=1.531$ $(\lambda=0.633 \mu \mathrm{m}), n_{p}=n_{\perp} . y=\sin \theta_{s} \cos \varphi_{s}, z=\sin \theta_{s} \sin \varphi_{s},-8^{\circ} \leq \theta_{s} \leq 8^{\circ}, 0^{\circ} \leq \varphi_{s} \leq 360^{\circ}$. Illumination by linearly polarized plane wave with polarization angle $\alpha=0^{\circ}$.

Angular distributions of light scattered by droplet and monolayer under illumination by unpolarized radiation are shown in Fig. 11. The calculations are performed using relations (15), (22), (25). It can be seen that the asymmetry effect also occurs in this case.
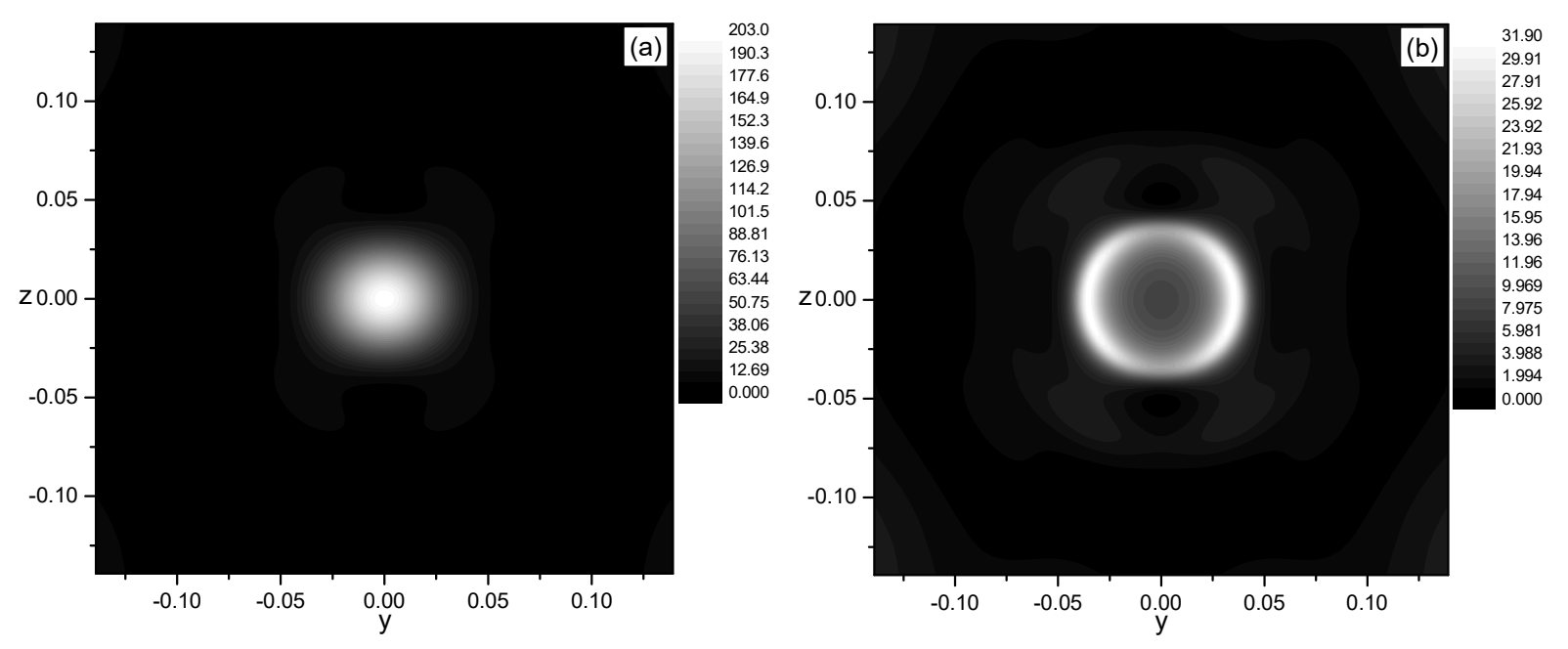

FIG. 11. Calculated $I_{n p}{ }^{s}\left(\theta_{s}, \varphi_{s}\right)$ scattering patterns for the single LC droplet (a), (c), (e) and scattering patterns $I_{n p}^{i n c}\left(\theta_{s}, \varphi_{s}\right)$ for the PDLC monolayer of monodisperse spherical droplets (b), (d), 
(f), illuminated by the unpolarized light. (a), (b): $w=0 \%$. (c), (d): $w=100 \%$. (e), (f): $w=50 \%$. $c=5$ $\mu \mathrm{m}, \eta=0.5, n_{\|}=1.717, n_{\perp}=1.531(\lambda=0.633 \mu \mathrm{m}), n_{p}=n_{\perp} . \quad y=\sin \theta_{s} \cos \varphi_{s}, z=\sin \theta_{s} \sin \varphi_{s}, \quad-8^{\circ} \leq \theta_{s} \leq 8^{\circ}$, $0^{\circ} \leq \varphi_{s} \leq 360^{\circ}$.

The effect of polydispersity of droplets and disorientation of their optical axes on the angular structure of the intensity of light scattered by the PDLC monolayer is illustrated by Fig. 12 for filling factor $\eta=0.3$.

The disorientation of the optical axes $\mathbf{N}_{j}$ of droplets [45] was modeled using uniform (with respect to the orientation angle $\left.\varphi_{d}\right)$ probability density distribution $P\left(\varphi_{d}\right)$.

$$
P\left(\varphi_{d}\right)=\left\{\begin{array}{l}
1 /\left(2 \varphi_{m}\right),\left|\varphi_{d}\right| \leq \varphi_{m} \\
0, \quad\left|\varphi_{d}\right|>\varphi_{m}
\end{array}\right.
$$

where $\varphi_{m}$ is the maximum deviation angle of the optical droplet axes relative to the $y$ axis of the laboratory coordinate system. At $\varphi_{m}=0^{\circ}$ droplet optical axes are oriented along the $y$ axis, at $\varphi_{m}=180^{\circ}$ they are oriented randomly in the plane of the monolayer. At $0^{\circ}<\varphi_{m}<180^{\circ}$ there is a partial orientation. Optical axes $\mathbf{N}_{j}$ of all droplets are in the monolayer plane.

The polydispersity of droplets was taken into account using the gamma distribution [33] of probability density $P(c)$ of droplets over radius $c$ as follows:

$$
P(c)=\frac{\mu^{\mu+1}}{\Gamma(\mu+1)} \frac{c^{\mu}}{c_{m}^{\mu+1}} \exp \left(-\mu c / c_{m}\right),
$$

where $\mu$ is the parameter of the distribution, $\Gamma$ is the gamma function, and $c_{m}$ is the modal (most probable) droplet radius. The modal radius $c_{m}$ and parameter $\mu$ are connected with the mean value $\langle c\rangle$ of the droplet radius and variation coefficient $D_{c} /\langle c\rangle$, where $D_{c}$ is the standard (mean-square) deviation, as follows:

$$
\begin{aligned}
& c_{m}=\frac{\mu}{\mu+1}\langle c\rangle, \\
& \mu=1 /\left(D_{c} /\langle c\rangle\right)^{2}-1 .
\end{aligned}
$$

FIG. 12. Calculated $I_{v v}{ }^{i n c}\left(\theta_{s}, \varphi_{s}\right)(\mathrm{a}, \mathrm{b})$ and $I_{v h}{ }^{i n c}\left(\theta_{s}, \varphi_{s}\right)(\mathrm{c}, \mathrm{d})$ scattering patterns for PDLC monolayer of polydisperse droplets. $(\mathrm{a}, \mathrm{c})$ : optical axes of droplets are oriented along the $y$ axis $\left(\varphi_{m}=0^{\circ}\right)$. $(\mathrm{b}, \mathrm{d})$ : optical axes of droplets are oriented randomly in the monolayer plane $(y, z)\left(\varphi_{m}=180^{\circ}\right)$. Modal radius 
of droplets $c_{m}=5 \mu \mathrm{m}$. Variation coefficient $D_{c} /\langle c\rangle=0.2 . w=50 \%, \alpha=0^{\circ}, \eta=0.3, n_{\|}=1.717, n_{\perp}=1.531$ $(\lambda=0.633 \mu \mathrm{m}), n_{p}=n_{\perp}, y=\sin \theta_{s} \cos \varphi_{s}, z=\sin \theta_{s} \sin \varphi_{s},-8^{\circ} \leq \theta_{s} \leq 8^{\circ}$ and $0^{\circ} \leq \varphi_{s} \leq 360^{\circ}$.

As one can see from Fig. 12, the polydispersity of droplets and disorientation of their optical axes change the asymmetry effect manifestation but do not lead to the effect disappearance.

The asymmetry effect arises owing to inhomogeneity in the anchoring of liquid crystal molecules at the droplet surface. The effect has an interference nature and can be explained on the basis of the Huygens-Fresnel principle. In a droplet with inhomogeneous surface anchoring, the distributions of the local refractive indices in regions corresponding to tangential and normal anchoring (Fig. 3) are different. As a consequence, the phase differences for the secondary waves propagating from these regions in the $+\theta_{s}$ and $-\theta_{s}$ directions are different. Interference of these waves at angles $+\theta_{s}$ and $-\theta_{s}$ results in different values of the scattered light intensities. The asymmetry effect depends on concentration, size, shape of droplets, and illumination conditions.

\section{SMALL-ANGLE DISTRIBUTION OF LIGHT AT THE QUENCHING EFFECT EMPLEMENTATION}

Let us consider the asymmetry in the angular distribution of the scattered light intensity under conditions of the interference quenching effect implementation. In such a case the coherent component of transmitted light vanishes [33, 59 -62].

When monolayer of monodisperse LC droplets is illuminated by the linearly polarized plane wave along the normal to the layer, coherent transmission coefficient $T_{c}$ is determined as follows [33]:

$$
T_{c}=1-Q \eta+\frac{Q^{2} L}{2} \eta^{2}
$$

where

$$
Q=\frac{4 \pi}{k^{2} \sigma} \operatorname{Re} f_{v v}\left(\mathbf{k}_{s}=\mathbf{k}_{i}\right)
$$

is the extinction efficiency factor of a LC droplet. Parameter

$$
L=\frac{1}{2}\left(1+\frac{\operatorname{Im}^{2} f_{v v}\left(\mathbf{k}_{s}=\mathbf{k}_{i}\right)}{\operatorname{Re}^{2} f_{v v}\left(\mathbf{k}_{s}=\mathbf{k}_{i}\right)}\right)\left(1+\frac{\left|f_{v h}\left(\mathbf{k}_{s}=\mathbf{k}_{i}\right)\right|^{2}}{\left|f_{v v}\left(\mathbf{k}_{s}=\mathbf{k}_{i}\right)\right|^{2}}\right) .
$$

When $L=0.5$ and $\eta=2 / \mathrm{Q}$, the value of $T_{c}=0$. It means that the coherently (directly) transmitted light is absent. We have quenching effect. It consists in redistribution of scattered light from the scattering direction $\theta_{s}=0$ to scattering angles $\theta_{s} \neq 0$. The realization of these conditions depends $[33,60-62]$ on the refractive indices of the polymer, liquid crystal, concentration of LC droplets and the polarization angle $\alpha$ of the incident light. 
The coherent transmission coefficient $T_{c}$ of the monolayer and $I_{v v}{ }^{i n c}$ component of the scattered light intensity is shown in Fig.13. The calculations are performed for LC with refractive indices $n_{\perp}=1.5, n_{\|}=1.7$, refractive index of polymer $n_{p}=1.56(@ \lambda=0.633 \mu \mathrm{m})$, and $w=50 \%$. Figure 13a illustrates the dependence of $T_{c}$ on the size parameter $\rho=2 \pi c n_{p} / \lambda$, where $c$ is the radius of the droplets. Figure 13b shows the data for $I_{\nu v}{ }^{i n c}\left(\theta_{s}\right)$ components at different azimuthal scattering angles $\varphi_{\mathrm{s}}$. The used parameters $\left(n_{p}=1.56, c=1.91 \mu \mathrm{m}\right.$, and $\left.\eta=0.713\right)$ are chosen so that at $\alpha=0^{\circ}$ the quenching effect is emplemented.
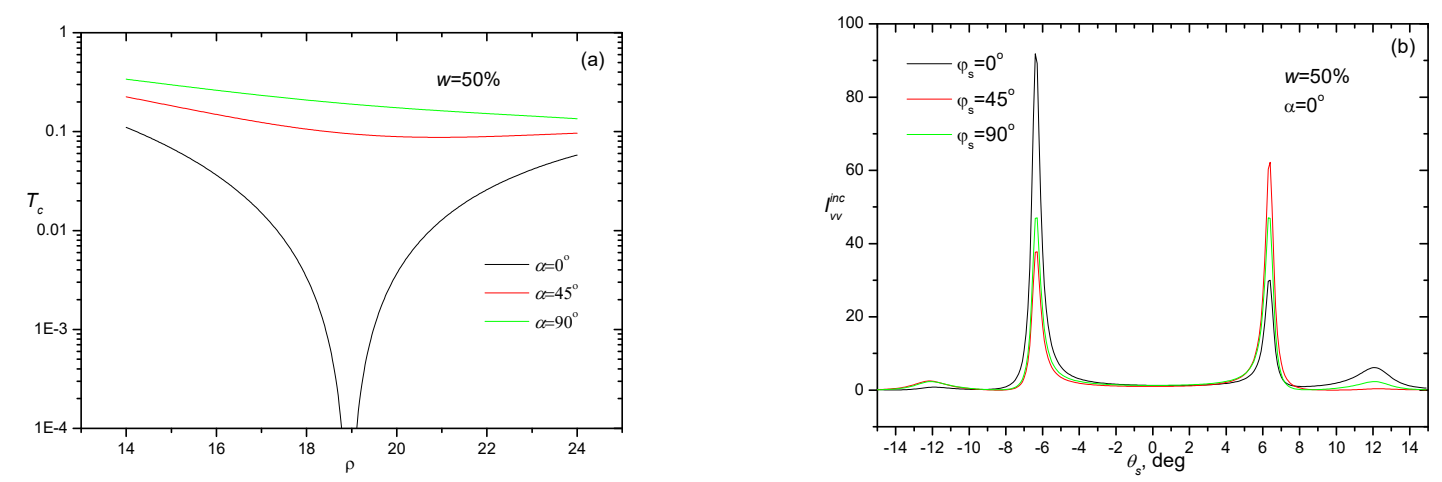

FIG. 13. Coherent transmission coefficient $T_{c}$ of the monolayer as function of the size parameter $\rho$ for different $\alpha$-values (a) and the $I_{v v}^{i n c}\left(\theta_{s}\right)$ component of the scattered light intensity at $\alpha=0^{\circ}$ for different $\varphi_{s}$-values (b). $n_{\perp}=1.5, n_{\|}=1.7, \lambda=0.633 \mu \mathrm{m}, n_{p}=1.56, c=1.91 \mu \mathrm{m}, w=50 \%, \eta=0.713$.

From data presented in Fig $13 \mathrm{~b}$ and Fig. 8 one can see that the quenching effect results in more pronounced assymetry effect.

\section{EXPERIMENT}

The $I_{v v}{ }^{i n c}\left(\theta_{s}, \varphi_{s}\right)$ and $I_{v h}{ }^{i n c}\left(\theta_{s}, \varphi_{s}\right)$ intensity components of scattered light were measured for PDLC film based on the 5CB nematic LC dispersed in the polyvinyl alcohol (PVA) plasticized with glycerine (Gl). Nematic was previously doped with ionic surfactant cetyltrimethylammonium bromide (CTAB). The weight ratio of PDLC components was: LC:CTAB:PVA:Gl=1:0.040:2.653:0.757. The samples were prepared by the emulsification method [1]. The refractive indices of $5 \mathrm{CB}$ measured at $t=23{ }^{\circ} \mathrm{C}$ and $\lambda=0.633 \mu \mathrm{m}$ are $n_{\perp}=1.531$ and $n_{\|}=1.717$. The refractive index of the polymer matrix is $n_{\mathrm{p}}=1.503$. PDLC film was formed on the glass substrate with ITO electrodes separated by the gap. Such cell design allows applying AC electric field along the film plane. 
The scattering patterns were obtained using the optical setup [45] with the $\mathrm{He}-\mathrm{Ne}$ laser LASOS $(\lambda=0.633 \mu \mathrm{m})$. The light passing through the polarizer normally incidents on surface of the PDLC sample. The scattered light was registrated using the analyzer.

Thickness of the tested PDLC film was $13.0 \pm 0.5 \mu \mathrm{m}$. The sample contained spheroidal droplets with the radial structure (parameter $w=100 \%$ ). Avearge droplet radius in the film plane was $3.25 \mu \mathrm{m}$ and the variation coefficient $D_{c} /\langle c\rangle=1.35$. The film thickness and the droplet oblateness were determined by observation [63] of the cross section of the sample in optical microscope. The average ratio $\varepsilon$ of minor axis of spheroidal droplets to the major one was equal $0.49 \pm 0.05$. The layer filling factor was $\eta=0.22$. Under the DC voltage $\mathrm{U}$, the tangential boundary conditions were formed at the part of droplet surface. At $U=27 \mathrm{~V}$ the average ratio of height of the segment with homeotropic anchoring to the droplet diameter $w=70 \%$. The droplet optical axes are oriented along to the applied electric field.

The photographs of scattering patterns of polarized laser radiation by ensemble of radial droplets $(w=100 \%)$ and ensemble of droplets with inhomogeneous anchoring $(w=70 \%)$ are presented in Fig. 14.
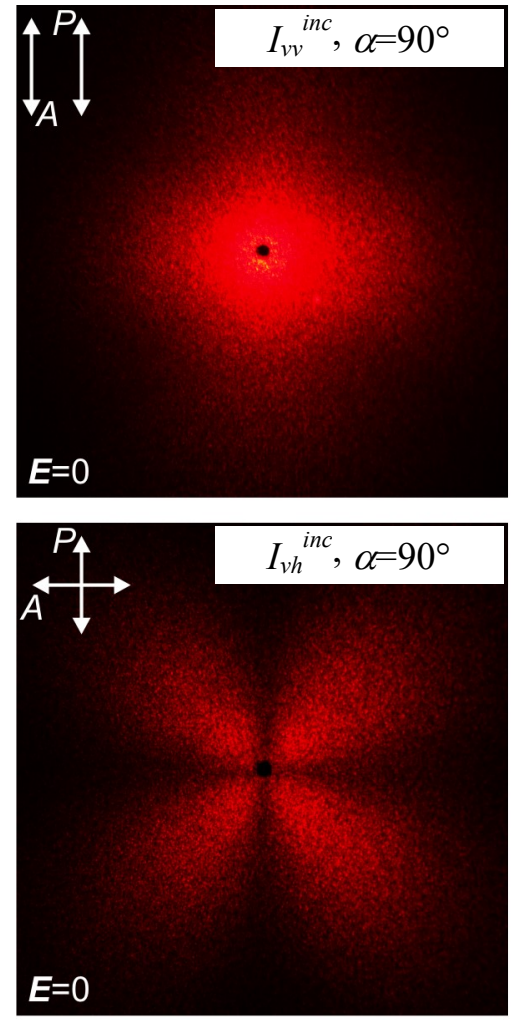

(a)
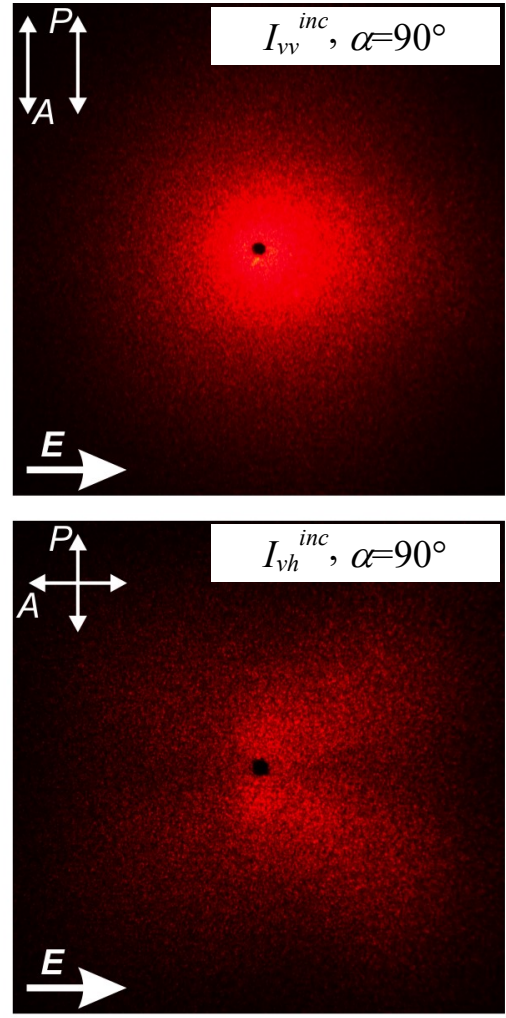

(b)
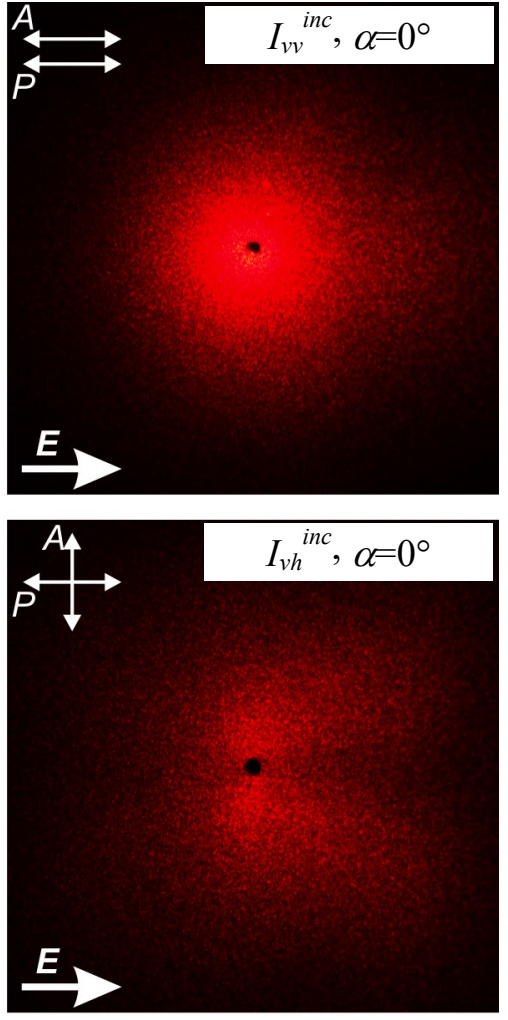

(c)

FIG. 14. Photographs of the scattering patterns of linearly polarized laser radiation made in off- $(a)$ and on-state of the electric field (constant voltage of $27 \mathrm{~V}$ was applied) $(b),(c)$. The photographs 
are made in parallel (top row) and crossed (bottom row) polarizers. The direction of the polarizer is perpendicular $(b)$ and parallel $(c)$ to the direction of the applied field. The central spot of the directly transmitted beam is shaded. The directions of the polarizer $(P)$ and the analyzer $(A)$ are indicated by double arrows, the direction of the applied field is designated by single arrows.

It can be seen that the pattern of light scattered by droplets with radial configuration is symmetric (Fig. 14a), while the pattern of light scattered by droplets with inhomogeneous anchoring is asymmetric (Fig. 14b, c).

\section{CONCLUSION}

The methods to describe and numerically simulate the small-angle distribution of radiation scattered by a PDLC monolayer with inhomogeneous surface anchoring have been developed. They are based on the anomalous diffraction approximation and the interference approximation. The results illustrate the change in the structure of the radiation scattered at small angles as a function of the internal structure of LC droplets, their concentration and polydispersity. The effect of the orientation angle of the scattering plane on the intensity of the scattered radiation is investigated. The symmetry breaking of the small-angle distribution of the scattered light intensity is analyzed as a function of size, polydispersity and disorientation of optical axes of liquid crystal droplets. The model developed makes it possible to analyze not only small-angle part of the scattered radiation but also the total scattering diagram, if instead of the anomalous difraction approach, one uses another approaches, for example, the Wentzel-Kramers-Brillouin approximation [27] to describe scattering by an individual LC droplet.

The obtained results can be used at development of electro-optical devices based on the liquid crystal dispersions (amplitude and phase modulators of light, polarizers and light-state transducers, displays, etc.). In particular, the symmetry breaking effect in angular distribution of scattered light can be used to develop devices for masking the display information. We have to note that stronger asymmetry effect can be achieved in the films with microfluidics generated LC droplets [64-67], because they have narrow size distribution of droplets and the filling factor of these films can be essentially higher than in the PDLC films.

\section{ACKNOWLEDGMENTS}

This work was performed under the interacademic integration project between the National Academy of Sciences of Belarus and the Siberian Branch of the Russian Academy of Sciences and 
supported by the Belarusian Republican Foundation for Fundamental Research (project no. F18RA003).

\section{References}

1. P. S. Drzaic, Liquid Crystal Dispersions (World Scientific, Singapore, 1995), p. 430.

2. F. Simoni, Nonlinear Properties of Liquid Crystals and Polymer Dispersed Liquid Crystals (World Scientific, Singapore, 1997), p. 259.

3. Handbook of Visual Display Technology, edited by J. Chen, W. Cranton, M. Fihn (Springer International Publishing, Switzerland, 2016) p. 3523.

4. Robert H. Chen, Liquid Crystals Displays: Fundamental Physics and Technology (John Wiley \& Sons, Inc. Wiley, 2011) p. 520.

5. V. Ya. Zyryanov, S. L. Smorgon, V. F. Shabanov, Molecular Engineering 1, 305 (1992).

6. F. Basile, F. Bloisi, L. Vicari, and F.Simoni, Phys. Rev. E. 48, 432 (1993).

7. V. V. Presnyakov, T. V. Galstian, Mol. Cryst. Liq.Cryst. 413, 435 (2004).

8. V. A. Loiko, A. V. Konkolovich, JETP 96, 489 (2003).

9. V. A. Loiko, A. V. Konkolovich, JETP 99, 343 (2004).

10. V. A. Loiko, A. V. Konkolovich, JETP 103, 935 (2006).

11. P. G. Lisinetskaya, A. V. Konkolovich, A. V. Loiko, Appl. Opt. 48, 3144 (2009).

12. A. Khan, I. Shiyanovskaya, T. Schneider, E. Montbach, D. J. Davis, N. Miller, D. Marhefka, T. Ernst, F. Nicholson, J. W. Doane, Journal of the SID. 15, 9 (2007).

13. V. A. Loiko, in Nanodroplets, edited by Z. M. Wang (Springer, New York, Heidelberg, Dordrecht, London, 2013), Chap. 9, p. 195-235.

14. Vandna Sharma, Pankaj Kumar, Amit Sharma, Chinky, Kuldeep Kumar Raina, J. of Mol. Liq. 233, 122 (2017).

15. L. H. Ye, C. S. Lv, F. J. Li, Y. Wang, B. Liu, Y. P Cui, J. Mod. Opt. 64, 14 (2017).

16. S. J. Kłosowicz, M. Aleksander, Opt.-Electr. Rev. 12, 305 (2004).

17. G. E. Volovik, O. D. Lavrentovich, Г. Е. Воловик, JETP 58, 1159 (1983).

18. J. L. West, J. W. Doane, S. Zumer, Patent US 4.685.771. Int.Cl. G02F 1/13 (Publ. 11.08.1987).

19. V. K. Freedericksz, V. Zolina, Trans. Far. Soc. 29, 919 (1933).

20. E. Dubois-Violette, P.G. De Gennes, J. de Phys. Lett. 36, L-255 (1975).

21. L. M. Blinov, E. I. Kats, and A. A. Sovin, Sov. Phys. Usp. 30, 604 (1987).

22. V. Ya. Zyryanov, M. N. Krakhalev, O. O. Prishchepa, and A. V. Shabanov. JETP Lett. 86, 383 (2007).

23. S. Zumer, J. W. Doane, Phys. Rev. A. 34, 3373 (1986). 
24. S. Zumer, Phys. Rev. A. 37, 4006 (1988).

25. D. A. Yakovlev and O. A. Afonin, Opt. and Spectr. 82, 78 (1997).

26. V. A. Loiko, P. G. Maksimenko, A. V. Konkolovich, Opt. and Spectrosc. 105, 791 (2008).

27. V. A. Loiko, A. V. Konkolovich, A. A. Miskevich, JETP 122, 176 (2016).

28. V. A. Loiko, U. Maschke, V. Ya. Zyryanov, A. V. Konkolovich, A. A. Miskevich, Opt. and Spectrosc. 120, 143 (2016).

29. V. A. Loiko, A. V. Konkolovich, V. Ya. Zyryanov, A. A. Miskevich, JETP 124, 388 (2017).

30. V. A. Loiko, V. Ya. Zyryanov, U. Maschke, A. V. Konkolovich, A. A. Miskevich, J. Qunt. Spectrosc. Radiat. Transfer. 113, 2585 (2012).

31. V. A. Loiko, V. Ya. Zyryanov, A. V. Konkolovich, A. A. Miskevich, M. N. Krakhalev, U. Maschke, Mol. Cryst. Liq. Cryst. 561, 194 (2012).

32. V. Yu. Rudyak, A. V. Emelyanenko, V. A. Loiko, Phys. Rev. E. 88, 052501 (2013).

33. V. A. Loiko, A. V. Konkolovich, J. Phys. D: Appl. Phys. 33, 2201 (2000).

34. A. Ishimary, Wave Propadation and Scattering in Random Media (Academic, New York, San Francisco, London, 1978; Mir, Moscow, 1986), Vol. 1, 2, p. 572.

35. M. Born and E. Wolf, Principles of Optics (Pergamon, Oxford, 1964; Nauka, Moscow, 1973), p. 720.

36. C. Bohren and D. Huffman, Absorption and Scattering of Light by Small Particles (Wiley, New York, 1998; Mir, Moscow, 1986), p. 531.

37. J. Ziman, Models of Disorder: The Theoretical Physics of Homogeneously Disordered Systems (Cambridge Univ., Cambridge, New York, 1979; Mir, Moscow, 1982), p. 591.

38. K. H. Ding, C. E. Mandt, L. Tsang, J. A. Kong, J. of Electromagnetic Waves and applications 6, 1015 (1992).

39. K. M. Hong, J. Opt. Soc.Am. 70, 821 (1980).

40. Scattering of electromagnetic waves: Numerical Simulations, edited By J. Kong (J. Wiley \& Sons, New York, 2001), p. 736.

41. Y. Rosenfeld, Phys. Rev. A. 42, 5978 (1990).

42. J. A. Lock, Chiu Chin-Lien, Appl. Opt. 33, 4663 (1994).

43. V. A. Loiko and A. V. Konkolovich, Opt. and spectrosc. 85, 568 (1998).

44. V. A. Loiko, U. Maschke, V. Ya. Zyryanov, A. V. Konkolovich, A. A. Miskevich, Opt. and Spectrosc. 110, 110 (2011).

45. V. A. Loiko, M. N. Krakhalev, A. V. Konkolovich, O. O. Prishchepa, A. A. Miskevich, V. Ya. Zyryanov, J. Qunt. Spectrosc. Radiat. Transfer. 178, 263 (2016).

46. H. C. van de Hulst, Light Scattering by Small Particles (Dover, New York, 1981), p. 470. 
47. G. H. Meeten, Optica Acta. 29, 759 (1982).

48. R. Azzam and N. Bashara, Ellipsometry and Polarized Light (North-Holland, Amsterdam, 1977; Mir, Moscow, 1981), p. 583.

49. E. V. Ishchenko and A. L. Sokolov, Polarization Optics (Mosk. Energet. Inst., Moscow, 2005 [in Russian]), p.454.

50. L. M. Blinov, Structure and properties of liquid crystals (Springer, Dordrecht, Heidelberg, London, New York, 2011).

51. O. O. Prishchepa, A.V. Shabanov, V.Ya. Zyryanov, Phys. Rev. E., 72, 031712 (2005).

52. V. Ya. Zyryanov, M. N. Krakhalev, O. O. Prishchepa, Mol. Cryst. Liq. Cryst. 489, 273 (2008).

53. V. Ya. Zyryanov, M. N. Krakhalev, O. O. Prishchepa, and A. V. Shabanov. JETP Letters. 88, 597 (2008).

54. A. Walther, A. Muller, Soft Matter. 4, 663 (2008).

55. A. Perro, S. Reculusa, S. Ravaine, E. Bourgeat-Lami, E. Duguet, J. Mater. Chem. 15, $3745(2005)$.

56. A. Waltherand, and A. H. E. Mueller, Chem. Rev., 113, 5194 (2013).

57. C.-C. Lin, C.-W. Liao, Y.-C. Chao, and C. Kuo, ACS Appl. Mater. Interfaces 2, 3185 (2010).

58. W. Lu, M. Chen, and L. Wu, J. Colloid Interface Sci., 328, 98 (2008).

59. V. A. Loiko, V. P. Dick, A. P. Ivanov, J.Opt. Soc. Am.A. 17, 2040 (2000).

60. V. A. Loiko, A. A. Miskevich, in Springer Series in Light Scattering, Vol. 1: Multiple Light Scattering, Radiative Transfer and Remote Sensing, edited by A. Kokchanovsky (Springer International Publishing AG Vitrociset, Belgium, Darmstadt, Germany, 2018), Chap. 2, p. 101-230.

61. V. A. Loiko, A. V. Konkolovich, Mol. Cryst. Liq. Cryst. 351, 371 (2000).

62. A. V. Konkolovich, V. V. Presnyakov, V. Ya. Zyryanov, V. A. Loiko, and V. F. Shabanov JETP Lett. 71, 486 (2000).

63. O. O. Prishchepa, A. V. Shabanov, V. Ya. Zyryanov, A. M. Parshin, V. G. Nazarov, JETP Lett. 84, 723 (2006).

64. D. E. Lucchetta, F. Simoni, P. Pagliusi, and G. Cipparrone, Opt. Data Process. Storage 1, $16(2015)$.

65. D. E. Lucchetta, F.Simoni, R.J.Hernandez, A.Mazzulla, and G. Cipparrone, Mol. Cryst. Liq. Cryst. 649, 11 (2017).

66. A. Mazzulla, G. Cipparrone, R. J. Hernandez, A. Pane, R. Bartolino, Mol. Cryst. Liq. Cryst. 576, 15 (2013). 
67. D. Rudhardt, A. Ferna'ndez-Nieves, D. R. Link, and D. A. Weitz, Appl. Phys. Lett. 82, $2610(2003)$. 